\title{
Extreme sports performance for more than a week with severely fractured sleep
}

\author{
Nikolaus C. Netzer ${ }^{1,2,3,4}$ (D) Linda K. Rausch ${ }^{2} \cdot$ Hannes Gatterer $^{3} \cdot$ Martin Burtscher $^{2} \cdot$ Arn H. Eliasson $^{5}$. \\ Stephan Pramsohler ${ }^{1}$
}

Received: 11 May 2020 / Revised: 10 August 2020 / Accepted: 14 August 2020 / Published online: 10 September 2020

(C) The Author(s) 2020

\begin{abstract}
Purpose Severely fractured sleep is mostly portrayed negatively, but investigations in extreme sports show that humans can maintain performance with a minimum of sleep. With two cases of long-lasting extreme sports performances, we demonstrate that severely fragmented sleep does not necessarily lead to a deterioration of physical and cognitive performance.

Methods We performed continuous polysomnography on a 34 year-old skier for 11 days and nights during a world record attempt in long-term downhill skiing and monitored a 32 year-old cyclist during the Race Across America for 8.5 days via sleep and activity logs.

Results The skier slept fractured fashion in 15-16 naps with a daily average of $6 \mathrm{~h}$ consisting of $77 \%$ in sleep stage 1 and $2,11 \%$ in stage 3, and $13 \%$ in stage REM. The cyclist slept a total of $7 \mathrm{~h}$ and $52 \mathrm{~min}$ in 8.5 days, split up into 11 short naps and 6 sleep periods. The average duration of napping was $8.8 \mathrm{~min}$ and of sleep $64.2 \mathrm{~min}$.

Conclusions These two cases demonstrate that outstanding performances are possible with severely fractured sleep and/or sleep deprivation. In well-trained athletes, breaking new recordsis possible despite extreme sleep habits.
\end{abstract}

Keywords Skiing $\cdot$ Bicycling $\cdot$ Extreme sleep fragmentation $\cdot$ Sleep diary $\cdot$ Polysomnography

\section{Introduction}

Under extreme conditions, humans are capable of performing on a high physical and mental level despite disrupted sleep or sleep deprivation, namely, in professional extreme sports events [1-5]. This observation has recently gained popular attention due to articles in the National Geographic

Nikolaus C. Netzer

nikinetzer@yahoo.com

1 Hermann Buhl Institute for Hypoxia and Sleep Medicine Research, University Innsbruck, Austria, Bad Aibling, Germany

2 The Institute of Sport Science, University Innsbruck, Innsbruck, Austria

3 Institute of Mountain Emergency Medicine, Eurac Research, Bozen, Italy

4 Department of Medicine, Division of Sport Medicine and Rehabilitation, University Hospitals, Ulm, Germany

5 Department of Medicine, Uniformed Services University of the Health Sciences, Bethesda, MD, USA
Magazine [6]. However, it has been well established that continuous sleep fragmentation serves as a stressor for athletes in training [7, 8], and it can consequently lead to poor athletic performance as well as to mental health symptoms or disorders [9]. Although many elite athletes are frequently traveling through different time zones, a dysregulation of the circadian rhythm in the long-term is dangerous, because it can impair not only performance but also metabolism and psychological functions [9].

However, there is limited published information about the effect of disrupted sleep or sleep fragmentation on endurance racing performances of extreme sports athletes [10]. One of the few studies collecting polysomnographic data on voluntary sleep fragmentation during extreme athletic performance was conducted by Edinger et al. (2010), who monitored the sleep of two individuals during a continuous tennis match lasting for $146 \mathrm{~h}$. Although the two athletes suffered from severe sleep restriction, they continued to obtain their usual amounts of slow wave sleep (SWS) throughout the marathon match [11]. Furthermore, Leger et al. (2008) assessed polysomnography (PSG) data of sailors competing in an 18day continuous yacht race. The data showed that sleepiness 
during the race depended upon depth of sleep before the race [12].

The purpose of this article is to provide further information about the effect of sleep fragmentation during endurance racing performance of extreme sports athletes.

\section{Methods}

\section{Marathon downhill skier}

We performed continuous PSG by the 2007 protocol of the American Academy of Sleep Medicine (AASM), manually scored by a single individual without respiratory channels in a healthy 34 year-old man for 11 days and nights during continuous downhill skiing. Sensors for leg EMG, oro-nasal airflow, and respiratory belts were not utilized, due todiscomfort of the athlete. The focus was set on the assessment of total sleep time (TST) and sleep stages; therefore EEG, EMG, and EOG data were collected. Sleep stages were defined as follows: $A w a k e=$ constant $\beta$ and $\alpha$ rhythm $(8-30 \mathrm{~Hz}$, amplitude $6-120 \mu \mathrm{V})$ in the EEG, stage 1 and $2=\theta$ rhythm $(20-100 \mu \mathrm{V}$, kcomplex in 2), stage $3=\delta$ rhythm $(0.5-3 \mathrm{~Hz}, 5-250 \mu \mathrm{V}$, $>20 \% \delta$ ), and stage REM = mainly $\theta$ rhythm (rapid eye movement in the EOG). The skiing activity contained cycles of 10 to $20 \mathrm{~min}$ of fast downhill skiing (speed > $50 \mathrm{~km} / \mathrm{h}$ ) on the slopes of Obergurgl in the Tyrolian Alps with 15-20 min lift rides giving an opportunity for napping and eating. In addition, every $48 \mathrm{~h}$ there was a 2$\mathrm{h}$ break for eating and massage. A team of physicians, physiotherapists, sleep technicians, and other trained skiers maintained continuous monitoring of the subject.

\section{RAAM Marathon cyclist}

We monitored a 32 year-old man during the "Race Across America" (RAAM) using sleep/activity logs. RAAM participants perform the distance of $4828 \mathrm{~km}$ including a climb over 53,340 $\mathrm{m}$ in one single stage over approximately 9 days. The athlete took sleep periods of approximately $60 \mathrm{~min}$ and naps of approximately $10 \mathrm{~min}$ whenever necessary. The exact time point of sleep periods/naps was decided during the race depending on the athlete's physical and emotional condition, state of exhaustion, weather, and course of the road. Both athletes were interviewed by an experienced sleep physician regarding their subjective sleep quality and the length of time required to return to normal cycles of daytime wakefulness and nighttime sleep 2 weeks after the extreme sports performances. Both athletes gave written consent (including publication of pictures), and the protocol was approved by the University of Innsbruck review board. Both athletes were examined by a physician and did not show any irregularities in health parameters.

\section{Results}

\section{Severely fractured sleep in marathon downhill skier}

PSG data collection was possible for $97 \%$ of the record attempt. The first sleep interval occurred after $10 \mathrm{~h}$ of skiing. During the recording time, a total sleep time of $68 \mathrm{~h}$ and 43 min was scored. This corresponds to an average sleep time of $6 \mathrm{~h}$ and $6 \mathrm{~min}$ per $24 \mathrm{~h}$. These average sleep hours were distributed over $55 \mathrm{~min}$ in sleep stage $\mathrm{N} 1(17 \%), 3 \mathrm{~h}$ and $19 \mathrm{~min}$ in stage $\mathrm{N} 2(60 \%), 36 \mathrm{~min}$ of slow wave sleep (SWS, stage N3) (11\%), as well as $42 \mathrm{~min}$ in stage rapideye-movement (REM) sleep (13\%). The structure of sleep changed over time with increasing amounts of SWS, N3, and REM sleep during the first 5 days and then stabilizing. Distribution of sleep stages on different days is presented in Fig. 1. We observed the maintenance of a regular sleep stage distribution during the gondola rides and during the 2-h massage breaks. The skier accumulated approximately 15 to 16 sleep cycles per $24 \mathrm{~h}$. There was only one fall by the skier during the entire event.

\section{Severely fractured sleep in RAAM marathon cyclist}

Throughout the race, a total amount of $7 \mathrm{~h}$ and $52 \mathrm{~min}$ of sleep and napping was logged. The distribution of sleep during the race is presented in Fig. 2. Average duration of the eleven naps was $8.8 \mathrm{~min}$. Average duration of the six sleep periods was $64.2 \mathrm{~min}$. The athlete did not record any severe health issues or injuries throughout the RAAM.

Both athletes subjectively estimated their total sleep time for the first night after their events to be less than $3 \mathrm{~h}$. They estimated their sleep onset latency for the first night to be around $1 \mathrm{~h}$. They also reported frequent awakenings in the first two nights after the event. Both reported a 1-week recovery time to return to their regular circadian rhythms (Fig. 3).

\section{Discussion}

Compared with previous PSG studies in extreme sports athletes, the monitoring of the downhill skier is the longest continuous PSG documentation of the rhythm of recurring napping in any athlete [11, 12]. Apparently, the human body is able to adapt to the rhythm of recurring napping structure over several days [13]. In the case of the marathon downhill skier, his sleeping habit adapted to the predefined 20 min resting periods during gondola rides with a healthy proportion of 
Fig. 1 Progression of Sleep Stage Distribution in Marathon

Downhill Skiing over the

Duration of Twelve Days.

Legend: $y$-axis shows the time of day; $x$-axis shows the distribution of sleep stages during each day; $R E M$ rapid eye movement sleep; $N 1-N 3$ = non-REM sleep; $N 1=$ relaxed wakefulness; $N 2=$ light sleep; $N 3$ = deep sleep, slow wave sleep; classification according to the American Academy of Sleep Medicine (AASM)

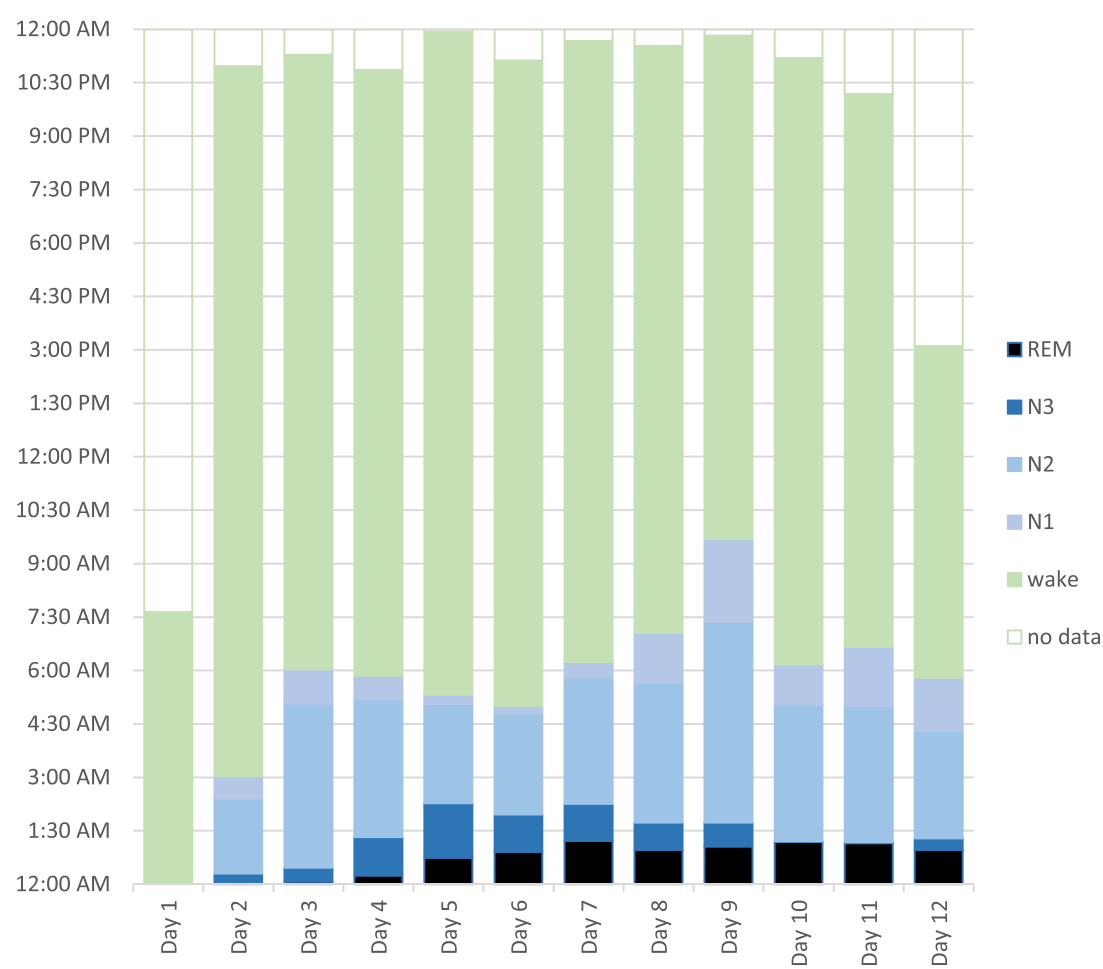

sleep phases resulting in reestablishing restorative functions. As an example of such sleep structure adaption in a nonsporting event, an indigenous population in South Africa, the Hazda tribe, demonstrates a similar ability to adapt sleep behavior during long distance hunts $[3,6]$.
Concerning the cyclist, this report is the first description of such a short total sleep time over a period greater than 8 days of extremely strenuous peak performance. In previous case studies, including another RAAM sleep report, total sleep times were longer by a multiple and with a different napping structure [1,14]. Lahart et al. (2013) report severe emotional
Fig. 2 Distribution of total sleep throughout the course of the Race Across America. Legend: $y$-axis shows the passages of time during 1 day; $x$-axis shows each day of the race; pre-/post-race defines the beginning of the race at $04: 18 \mathrm{PM}$ (GTM-7) and the finish point at 00:35 AM (GMT-4)

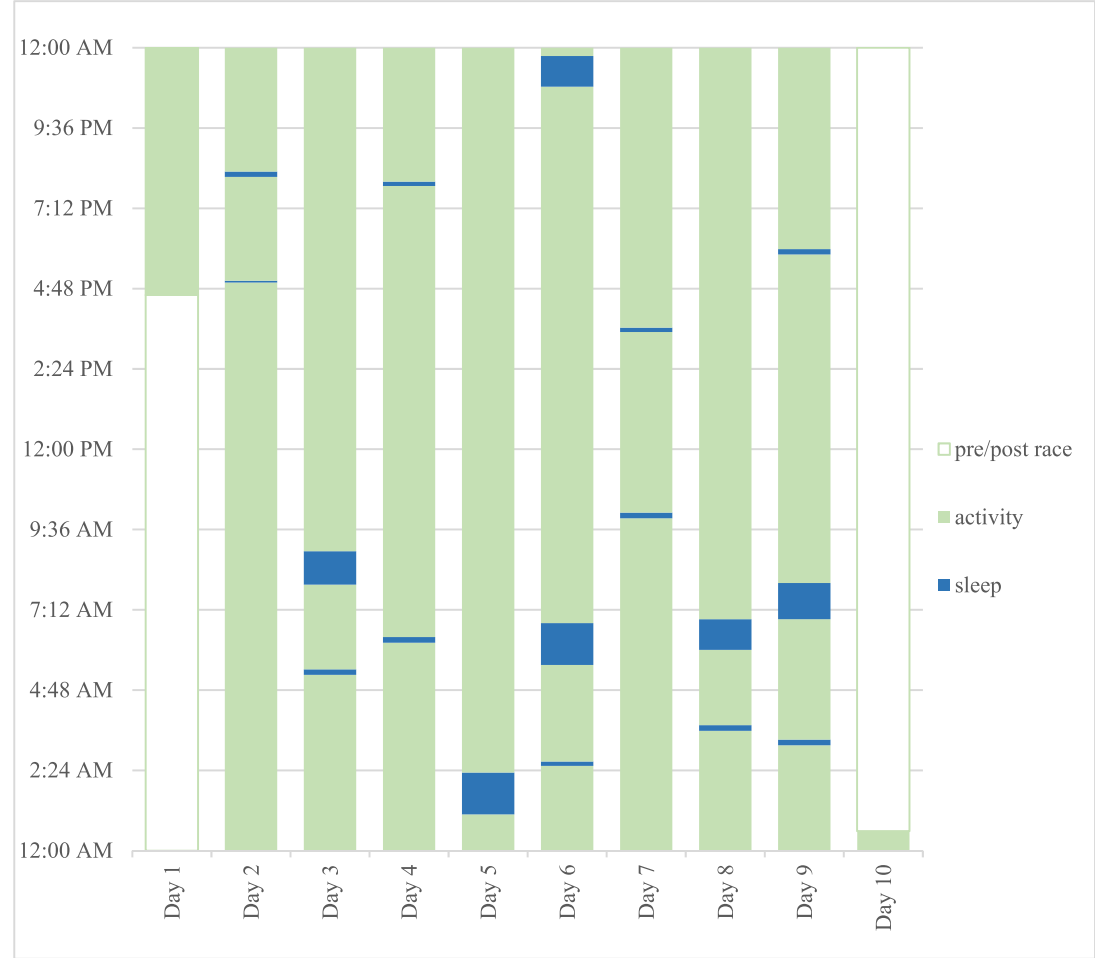


Fig. 3 The RAAM (Race Across America) 2015 winner's team member helping the athlete out of the bicycle saddle and into a comfortable napping position



impairment of other RAAM athletes during the event. Although the cyclist did not report such severe impairments, the long-term health effects of sleep deprivation during such extreme sports performances are not known and could be a risk for physical and mental well-being. The unique ability of the cyclist to withstand severe sleep restriction could be of further research interest; therefore it would be necessary to obtain PSG data in order to add information to the sleep/ activity logs.

As a limitation we acknowledge collection of PSG data in only one athlete. However, applying electrodes on the body of a cyclist who is performing such extreme exercise over such a long time period is not easily implemented. In comparison, we regard the application of PSG electrodes in the marathon skier as more feasible, because he had predefined resting periods due to gondola rides. A further limitation is that we do not have PSG or sleep-log data of normal nights outside of the extreme performance period. However, we expect the regular sleeping patterns in these extreme sports athletes to be ordinarily restorative; otherwise they would not be able to perform at such level.

These two cases demonstrate that outstanding performances, i.e., breaking a continuous ski record and winning the RAAM even while requiring high levels of concentration, are possible with severely fractured sleep and avoiding sleep phases much longer than 1 hour. Our observations contrast with previously described decriments in neuromuscular, hormonal, and cognitive functions after extreme performances of different types due to sleep deprivation and short continuous sleep periods [7, 8]. However, according to one of these prior publications, longer extreme sports events are associated with less neuromuscular fatigue than shorter events [8]. The latter finding may explain why the athletes described in this report could ski and cycle with good muscular control for more than a week.

\section{Conclusion}

We conclude from both cases of professional athletes that severely fragmented sleep in healthy well-trained athletes does not necessarily lead to a significant reduction of performance of a demanding but well-trained physical task. PSG recording demonstrated that if severely fractured sleep allows for a sufficient amount of sleep, the sleep stage distribution over each 24-h period reaches levels which are commonly seen in usual day-night sleep wake cycles.

However, possible health risks appearing from sleep deprivation, such as metabolism imbalances, mental disorders, or a negative long-term effect on performance cannot be disregarded. We suggest that extreme sports athletes should take care of a healthy sleep hygiene with the help of professionals in order to cope with possible negative effects.

Acknowledgments We would like to thank Mirko Gindulis MD for sleep data collection and evaluation in the marathon skier.

Funding Information Open access funding provided by University of Innsbruck and Medical University of Innsbruck.

\section{Compliance with ethical standards}

Conflict of interest Disclosure of potential conflict of interest: The authors declare that they have no conflict of interest.

Ethics approval The study protocol was reviewed and approved by the ethical review board of the University of Innsbruck in accordance with the ethical standards as laid down in the 1964 Declaration of Helsinki and its later amendments.

Informed consent Informed consent was obtained from both individual participants included in the study. Both individual participants gave written consent to publication of pictures. 
Open Access This article is licensed under a Creative Commons Attribution 4.0 International License, which permits use, sharing, adaptation, distribution and reproduction in any medium or format, as long as you give appropriate credit to the original author(s) and the source, provide a link to the Creative Commons licence, and indicate if changes were made. The images or other third party material in this article are included in the article's Creative Commons licence, unless indicated otherwise in a credit line to the material. If material is not included in the article's Creative Commons licence and your intended use is not permitted by statutory regulation or exceeds the permitted use, you will need to obtain permission directly from the copyright holder. To view a copy of this licence, visit http://creativecommons.org/licenses/by/4.0/.

\section{References}

1. Lahart IM, Lane AM, Hulton A et al (2013) Challenges in maintaining emotion regulation in a sleep and energy deprived state induced by the $4800 \mathrm{Km}$ ultra-endurance bicycle race; the race across AMerica (RAAM). J Sport Sci Med 12(3):481-488

2. Nindl BC, Billing DC, Drain JR, Beckner ME, Greeves J, Groeller H, Teien HK, Marcora S, Moffitt A, Reilly T, Taylor NAS, Young AJ, Friedl KE (2018) Perspectives on resilience for military readiness and preparedness: report of an international military physiology roundtable. J Sci Med Sport 21(11):1116-1124. https://doi.org/ 10.1016/j.jsams.2018.05.005

3. Samson DR, Crittenden AN, Mabulla IA, Mabulla AZP, Nunn CL (2017) Chronotype variation drives night-time sentinel-like behaviour in hunter-gatherers. Proc Biol Sci 284(1858):20170967. https://doi.org/10.1098/rspb.2017.0967

4. Tyyska J, Kokko J, Salonen M, Koivu M, Kyrolainen H (2010) Association with physical fitness, serum hormones and sleep during a 15-day military field training. J Sci Med Sport 13(3):356-359. https://doi.org/10.1016/j.jsams.2009.04.005

5. Zhang Y, Li Z, Liu X, Liu F, Jing X, Wu B (2015) Simulated spaceflight operations under sleep deprivation and confinement. Aerosp Med Hum Perform 86(10):865-874. https://doi.org/10. 3357/AMHP.4175.2015
6. Society NG (2018) The science of sleep. Washington DC:1-142

7. Hurdiel R, Pezé T, Daugherty J, Girard J, Poussel M, Poletti L, Basset P, Theunynck D (2015) Combined effects of sleep deprivation and strenuous exercise on cognitive performances during the north face ${ }^{\circledR}$ Ultra Trail du Mont Blanc $®$ (UTMB $\left.®\right)$ ). J Sports Sci 33(7):670-674. https://doi.org/10.1080/02640414.2014.960883

8. Saugy J, Place N, Millet GY, Degache F, Schena F, Millet GP (2013) Alterations of neuromuscular function after the world's most challenging mountain ultra-marathon. PLoS One 8(6):e65596. https://doi.org/10.1371/journal.pone.0065596

9. Reardon CL, Hainline B, Aron CM, Baron D, Baum AL, Bindra A, Budgett R, Campriani N, Castaldelli-Maia JM, Currie A, Derevensky JL, Glick ID, Gorczynski P, Gouttebarge V, Grandner MA, Han DH, McDuff D, Mountjoy M, Polat A, Purcell R, Putukian M, Rice S, Sills A, Stull T, Swartz L, Zhu LJ, Engebretsen L (2019) Mental health in elite athletes: International Olympic Committee consensus statement (2019). Br J Sports Med 53(11):667-699. https://doi.org/10.1136/bjsports-2019-100715

10. Reilly T, Edwards B (2007) Altered sleep-wake cycles and physical performance in athletes. Physiol Behav 90(2-3):274-284

11. Edinger JD, Marsh GR, McCall WV, Erwin CW, Lininger AW (1990) Daytime functioning and nighttime sleep before, during, and after a 146-hour tennis match. Sleep 13(6):526-532. https:// doi.org/10.1093/sleep/13.6.526

12. Leger D, Elbaz M, Raffray T, Metlaine A, Bayon V, Duforez F (2008) Sleep management and the performance of eight sailors in the tour de France a la voile yacht race. J Sports Sci 26(1):21-28. https://doi.org/10.1080/02640410701348636

13. Stampi C (1992) Evolution, chronobiology, and functions of polyphasic and ultrashort sleep: main issues. In: Stampi C (ed) Why we nap: evolution, chronobiology, and functions of polyphasic and ultrashort sleep. Birkhäuser, Boston, pp 1-20

14. Smith RS, Walsh J, Dement W (1998) Sleep deprivation and the race across America. Sleep (22):303-304

Publisher's note Springer Nature remains neutral with regard to jurisdictional claims in published maps and institutional affiliations. 